\title{
Kaehlerian manifolds with constant scalar curvature whose Bochner curvature tensor vanishes
}

By Kentaro YANO and Shigeru IshIHARA

\section{§1. Introduction}

Let $M$ be a Riemannian manifold of dimension $n \geqq 3$ and of class $C^{\infty}$. We cover $M$ by a system of coordinate neighborhoods $\left\{U ; x^{n}\right\}$, where here and in the sequel the indices $h, i, j, k, \cdots$ run over the range $\{1,2, \cdots, n\}$, and denote by $g_{j i}, \nabla_{i}, K_{k j i}{ }^{n}, K_{j i}$ and $K$ the positive definite metric tensor, the operator of covariant differentiation with respect to the Levi-Civita of $M$ connection, the curvature tensor, the Ricci tensor and the scalar curvature respectively.

A conformally flat Riemannian manifold is characterized by the vanishing of the Weyl conformal curvature tensor

$$
C_{k j i}{ }^{h}=K_{k j i}{ }^{h}+\delta_{k}^{h} C_{j i}-\delta_{j}^{h} C_{k i}+C_{k}{ }^{h} g_{j i}-C_{j}{ }^{h} g_{k i}
$$

and the tensor

$$
C_{k j i}=\nabla_{k} C_{j i}-\nabla_{j} C_{k i}
$$

where

$$
\begin{aligned}
C_{j i} & =-\frac{1}{n-2} K_{j i}+\frac{1}{2(n-1)(n-2)} K g_{j i}, \\
C_{k}{ }^{n} & =C_{k t} g^{t h} .
\end{aligned}
$$

Ryan [4] proved

THEOREM Let $M$ be a compact conformally flat Riemannian manifold with constant scalar curvature. If the Ricci tensor is positive semi-definite, then the simply connected Riemannian covering of $M$ is one of

$$
S^{n}(c), R \times S^{n-1}(c) \text { or } E^{n},
$$

the real space forms of curvature $c$ being denoted by $S^{n}(c)$ or $E^{n}$ depending on whether $c$ is positive or zero. (See also Aubin [1], Goldberg [3], Tani [6]).

He first proves that, in a conformally flat Riemannian manifold with constant scalar curvature $K$, we have 


$$
\begin{aligned}
\frac{1}{2} \Delta\left(K_{i h} K^{i h}\right) & =\frac{1}{2} g^{k j} \nabla_{k} \nabla_{j}\left(K_{i h} K^{i h}\right) \\
& =\frac{1}{n-2} P+\left(\nabla_{j} K_{i h}\right)\left(\nabla^{j} K^{i n}\right),
\end{aligned}
$$

where

$$
P=n K_{t}^{s} K_{s}^{r} K_{r}{ }^{t}-\frac{2 n-1}{n-1} K K_{j i} K^{j i}+\frac{1}{n-1} K^{3},
$$

and then that, if we denote by $\lambda_{i}(i=1,2, \cdots, n)$ the eigenvalues of $K_{j i}$, then we have

$$
P=\frac{1}{n-1} \sum_{i} \sum_{j \neq c} \lambda_{i}\left(\lambda_{i}-\lambda_{j}\right)\left(\lambda_{i}-\lambda_{k}\right) .
$$

He then assumes that the Ricci tensor $K_{j i}$ is positive semi-definite and shows that in this case we have $P \geqq 0$ on $M$.

Thus he obtains $\Delta\left(K_{i n} K^{i n}\right) \geqq 0$, from which

$$
P=0 \text { and } \nabla_{k} K_{j i}=0 \text {. }
$$

From these, he obtains the theorem quoted above. We can easily see that the conclusion of the theorem also applies if the assumptions of compactness and constant scalar curvature are replaced by local homogeneity of $M$.

The main purpose of the present paper is to prove the following theorem corresponding to that of Ryan, replacing the vanishing of the Weyl conformal curvature tensor in a Riemannian manifold by that of the Bochner curvature tensor in a Kaehlerian manifold.

THEOREM Let $M$ be a Kaehlerian manifold of real dimension $n$ with constant scalar curvature whose Bochner curvature tensor vanishes and whose Ricci tensor is positive semi-definite. If $M$ is compact, then the universal covering manifold is a complex projective spact $C P^{n / 2}$ or a complex space $C^{n / 2}$.

From the method of the proof we easily see that the conclusion of the theorem is also valid if the assumptions of compactness and constant scalar curvature are replaced by local homogeneity of $M$.

\section{§2. Preliminaries}

Let $M$ be a Kaehlerian manifold of real dimension $n$ and $(g, F)$ its Kaehlerian structure. That is, $g$ is a Riemannian metric and $F$ a complex structure in $M$ such that

$$
F_{j}{ }^{t} F_{i}^{s} g_{t s}=g_{j i}, \quad \nabla_{j} F_{i}^{h}=0,
$$


where $g_{j i}$ and $F_{i}^{h}$ are local components of $g$ and $F$ respectively. It is well known that

is skew-symmetric.

$$
F_{j i}=F_{j}{ }^{t} g_{t i}
$$

As a complex analogue to the Weyl conformal curvature tensor, Bochner [2] (see also, Yano and Bochner [9]) introduced the following curvature tensor in a Kaehlerian manifold:

$$
\begin{aligned}
B_{k j i}{ }^{h}= & K_{k j i}{ }^{n}+\delta_{k}^{h} L_{j i}-\delta_{j}^{h} L_{k i}+L_{k}{ }^{h} g_{j i}-\dot{L}_{j}{ }^{h} g_{k i} \\
& +F_{k}{ }^{n} M_{j i}-F_{j}{ }^{n} M_{k i}+M_{k}{ }^{n} F_{j i}-M_{j}{ }^{n} F_{k i} \\
& -2\left(M_{k j} F_{i}{ }^{n}+F_{k j} M_{i}{ }^{h}\right),
\end{aligned}
$$

where

$$
\begin{aligned}
L_{j i} & =-\frac{1}{n+4} K_{j i}+\frac{1}{2(n+2)(n+4)} K g_{j i}, \quad L_{k}{ }^{n}=L_{k t} g^{t h}, \\
M_{j i} & =-L_{j t} F_{i}^{t}=-\frac{1}{n+4} H_{j i}+\frac{1}{2(n+2)(n+4)} K F_{j i}, \\
M_{k}{ }^{h} & =M_{k t} g^{t h}, \quad H_{j i}=-K_{j t} F_{i}^{t} .
\end{aligned}
$$

Bochner introduced this curvature tensor using a complex local coordinate system. We call this curvature tensor the Bochner curvature tensor. The form (2.1) of the Bochner curvature tensor with respect to a real coordinate system has been given by Tachibana [5] (see also Yano [8]).

By a straightforward computation, we can prove

$$
\nabla_{t} B_{k j i}{ }^{t}=-n\left(\nabla_{k} L_{j i}-\nabla_{j} L_{k i}\right) \text {. }
$$

When the Bochner curvature tensor vanishes, we have, from (2.1),

$$
\begin{aligned}
K_{k j i h}= & \frac{1}{n+4}\left[g_{k h} K_{j i}-g_{j h} K_{k i}+K_{k h} g_{j i}-K_{j h} g_{k i}\right. \\
& \left.+F_{k h} H_{j i}-F_{j h} H_{k i}+H_{k h} F_{j i}-H_{j h} F_{k i}-2\left(H_{k j} F_{i h}+F_{k j} H_{i n}\right)\right] \\
& -\frac{K}{(n+2)(n+4)}\left[g_{k h} g_{j i}-g_{j h} g_{k i}+F_{k h} F_{j i}\right. \\
& \left.-F_{j h} F_{k i}-2 F_{k j} F_{i h}\right]
\end{aligned}
$$

for the covariant components $K_{k j i h}=K_{k j i}^{t} g_{t h}$ of the Riemannian curvature tensor.

\section{§3. Lemmas}

In this section, we prove some lemmas which will be used in the proof of the theorem. 
LEMMA 1. If the Bochner curvature tensor vanishes and the scalar curvature is constant in a Kaehlerian manifold, then we have

$$
\nabla_{k} K_{j i}-\nabla_{j} K_{k i}=0 \text {, }
$$

that is, $\nabla_{k} K_{j i}$ is a symmetric tensor.

Proof This follows from (2.3) and the definition of $L_{j i}$.

LEMMA 2. If the Bochner curvature tensor of a Kaehlerian manifold vanishes and the manifold is an Einstein manifold, then the Kaehlerian manifold is of constant holomorphic sectional curvature (see Tachibana [5]). PRoOF If the Bochner curvature tensor vanishes, we have, from (2.1),

$$
\begin{aligned}
K_{k j i}{ }^{n} & =-\delta_{k}^{h} L_{j i}+\delta_{j}^{h} L_{k i}-L_{k}{ }^{h} g_{j i}+L_{j}{ }^{h} g_{k i} \\
& -F_{k}{ }^{n}{ }_{j i} M+F_{j}{ }^{n} M_{k i}-M_{k}{ }^{n} F_{j i}+M_{j}{ }^{n} F_{k i}+2\left(M_{k j} F_{i}^{n}+F_{k j} M_{i}{ }^{n}\right) .
\end{aligned}
$$

If the manifold is an Einstein space, we have

$$
K_{j i}=\frac{K}{n} g_{j i}, \quad H_{j i}=\frac{K}{n} F_{j i},
$$

and consequently we have, from (2.2),

$$
L_{j i}=-\frac{K}{2 n(n+2)} g_{j i}, \quad M_{j i}=-\frac{K}{2 n(n+2)} F_{j i} .
$$

Thus, substituting these into (3.2), we find

$$
K_{k j i}{ }^{n}=\frac{K}{n(n+2)}\left[\delta_{k}^{h} g_{j i}-\delta_{j}^{h} g_{k i}+F_{k}{ }^{h} F_{j i}-F_{j}{ }^{h} F_{k i}-2 F_{k j} F_{i}{ }^{h}\right],
$$

which shows that the manifold is of constant holomorphic sectional curvature.

LemMa 3. In a Kaehlerian manifold of dimension $n$ with constant scalar curvature whose Bochner curvature tensor vanishes, we have

$$
\begin{aligned}
\frac{1}{2} \Delta\left(K_{i n} K^{i n}\right)= & \frac{1}{n+4}\left[n K_{t}^{s} K_{s}^{r} K_{r}^{t}-\frac{2(n+1)}{n+2} K K_{j i} K^{j t}+\frac{1}{n+2} K^{3}\right] \\
& +\left(\nabla_{j} K_{i n}\right)\left(\nabla^{j} K^{i n}\right) .
\end{aligned}
$$

Proof We first have

$$
\frac{1}{2} \Delta\left(K_{i n} K^{i n}\right)=g^{k j} \nabla_{k}\left[\left(\nabla_{j} K_{i n}\right) K^{i n}\right]=g^{k j}\left(\nabla_{k} \nabla_{j} K_{i n}\right) K^{i n}+\left(\nabla_{j} K_{i h}\right)\left(\nabla^{j} K^{i n}\right),
$$

that is,

$$
\frac{1}{2} \Delta\left(K_{i h} K^{i h}\right)=g^{k j}\left(\nabla_{k} \nabla_{h} K_{j i}\right) K^{i h}+\left(\nabla_{j} K_{i h}\right)\left(\nabla^{j} K^{i n}\right),
$$


where we have used (3.1) and $K_{i h}=K_{h i}$.

On the other hand, applying the Ricci formula to $K_{j i}$, we have

$$
\nabla_{k} \nabla_{h} K_{j i}=\nabla_{h} \nabla_{k} K_{j i}-K_{k h j}{ }^{t} K_{t i}-K_{k n i}{ }^{t} K_{j t} .
$$

Substituting this into (3.5), we obtain

$$
\begin{aligned}
\frac{1}{2} \Delta\left(K_{i h} K^{i n}\right)= & g^{k j}\left(\nabla_{h} \nabla_{k} K_{j i}-K_{k h j}{ }^{t} K_{t i}-K_{k n i}{ }^{t} K_{j t}\right) K^{i n} \\
& +\left(\nabla_{j} K_{i h}\right)\left(\nabla^{j} K^{i n}\right),
\end{aligned}
$$

that is,

$$
\frac{1}{2} \Delta\left(K_{i h} K^{i n}\right)=K_{t}^{s} K_{s}^{r} K_{r}^{t}-K_{k j i h} K^{k h} K^{j i}+\left(\nabla_{j} K_{i h}\right)\left(\nabla^{j} K^{i n}\right),
$$

where we have used (3.1) and $K=$ const.

We now compute $K_{k j i h} K^{k h} K^{j i}$. Using (2.4), we find

$$
\begin{aligned}
K_{k j i h} K^{k h} K^{j i}= & \frac{1}{n+4}\left[g_{k h} K_{j i}-g_{j h} K_{k i}+K_{k h} g_{j i}-K_{j h} g_{k i}\right. \\
& \left.-F_{j h} H_{k i}-H_{j h} F_{k i}-2\left(H_{k j} F_{i n}+F_{k j} H_{i h}\right)\right] K^{k h} K^{j i} \\
& -\frac{K}{(n+2)(n+4)}\left[g_{k h} g_{j i}-g_{j h} g_{k i}-F_{j h} F_{k i}\right. \\
& \left.-2 F_{k j} F_{i n}\right] K^{k h} K^{j i} .
\end{aligned}
$$

On the other hand, we have the following equalities:

$$
\begin{aligned}
& -F_{j h} H_{k i} K^{k n} K^{j i}=K_{k t} K_{h}{ }^{t} K^{k n}, \\
& -H_{j h} F_{k i} K^{k h} K^{j i}=K_{j t} K_{i}{ }^{t} K^{j i}, \\
& -H_{k j} F_{i h} K^{k n} K^{j i}=K_{k t} K_{h}{ }^{t} K^{k n}, \\
& -F_{k j} H_{i n} K^{k h} K^{j i}=K_{j}{ }^{t} K_{i t} K^{j i}, \\
& -F_{j h} F_{k i} K^{k n} K^{j i}=K_{j i} K^{j i}, \\
& -F_{k j} F_{i h} K^{k n} K^{j i}=K_{j i} K^{j i},
\end{aligned}
$$

where we have used $H_{j t}=-K_{j t} F_{i}^{t}$ and the identity

$$
F_{j}{ }^{t} F_{i}^{s} K_{t s}=K_{j i} \text {, }
$$

(see Yano [7]).

If we substitute these six equalities into (3.7), we have

$$
K_{k j t h} K^{k n} K^{j i}=\frac{1}{n+4}\left[K_{t}^{s} K_{s}^{r} K_{r}^{t}+\frac{2(n+1)}{n+2} K K_{j i} K^{j i}-\frac{1}{n+2} K^{3}\right] \text {. }
$$

Thus substituting this equation into (3.6), we have (3.4).

Lemma 4. (Ryan [4]) In a Riemannian manifold of dimension $n$, for 


$$
P=n K_{t}^{s} K_{s}^{r} K_{r}^{t}-\frac{2 n-1}{n-1} K K_{j i} K^{j i}+\frac{1}{n-1} K^{3},
$$

we have

$$
P=\frac{1}{n-1} \sum_{i} \sum_{j \neq k} \lambda_{i}\left(\lambda_{i}-\lambda_{j}\right)\left(\lambda_{i}-\lambda_{k}\right),
$$

where $\lambda_{1} \leqq \lambda_{2} \leqq \cdots \leqq \lambda_{n}$ are eigenvalues of the Ricci tensor $K_{j i}$.

Proof First we have

$$
\begin{aligned}
\sum_{i, j, k} \lambda_{i}\left(\lambda_{i}-\lambda_{j}\right)\left(\lambda_{i}-\lambda_{k}\right)= & \sum_{i, j, k}\left[\lambda_{i}{ }^{3}-\lambda_{i}{ }^{2} \lambda_{j}-\lambda_{i}{ }^{2} \lambda_{k}+\lambda_{i} \lambda_{j} \lambda_{k}\right] \\
= & \sum_{j, k}\left[K_{t}^{s} K_{s}^{r} K_{r}{ }^{t}-K_{t s} K^{t s} \lambda_{j}-K_{t s} K^{t s} \lambda_{k}\right. \\
& \left.+K \lambda_{j} \lambda_{k}\right] \\
= & \sum_{k}\left[n K_{t}^{s} K_{s}^{r} K_{r}{ }^{t}-K K_{t s} K^{t s}-n K_{t s} K^{t s} \lambda_{k}\right. \\
& \left.+K^{2} \lambda_{k}\right],
\end{aligned}
$$

that is,

$$
\sum_{i, j, k} \lambda_{i}\left(\lambda_{i}-\lambda_{j}\right)\left(\lambda_{i}-\lambda_{k}\right)=n^{2} K_{t}^{s} K_{s}{ }^{r} K_{r}^{t}-2 n K K_{t s} K^{t s}+K^{3} .
$$

Next we have

$$
\begin{aligned}
\sum_{i, j} \lambda_{i}\left(\lambda_{i}-\lambda_{j}\right)^{2} & =\sum_{i, j}\left[\lambda_{i}{ }^{3}-2 \lambda_{i}{ }^{2} \lambda_{j}+\lambda_{i} \lambda_{j}{ }^{2}\right] \\
& =\sum_{j}\left[K_{t}^{s} K_{s}{ }^{r} K_{r}{ }^{t}-2 K_{j i} K^{j i} \lambda_{j}+K \lambda_{j}{ }^{2}\right],
\end{aligned}
$$

that is,

$$
\sum_{i, j} \lambda_{i}\left(\lambda_{i}-\lambda_{j}\right)^{2}=n K_{t}^{s} K_{s}^{r} K_{r}{ }^{t}-K K_{j i} K^{j t} .
$$

From (3.10) and (3.11), we have

$$
\begin{aligned}
& \sum_{i} \sum_{j \neq k} \lambda_{i}\left(\lambda_{i}-\lambda_{j}\right)\left(\lambda_{i}-\lambda_{k}\right) \\
& =\sum_{i, j, k} \lambda_{i}\left(\lambda_{i}-\lambda_{j}\right)\left(\lambda_{i}-\lambda_{k}\right)-\sum_{i, j} \lambda_{i}\left(\lambda_{i}-\lambda_{j}\right)^{2} \\
& =n(n-1) K_{t}^{s} K_{s}^{r} K_{r}^{t}-(2 n-1) K K_{j i} K^{j i}+K^{3},
\end{aligned}
$$

which proves (3.9).

Lemma 5. In a Riemannian manifold of dimension $n$, for

$$
Q=n K_{t}^{s} K_{s}^{r} K_{r}{ }^{t}-\frac{2(n+1)}{n+2} K K_{j i} K^{j i}+\frac{1}{n+2} K^{3},
$$

we have

$$
Q=P+\frac{3 n}{(n-1)(n+2)} K\left(\left(K_{j i}-\frac{K}{n} g_{j i}\right)\left(K^{j i}-\frac{K}{n} g^{j i}\right) .\right.
$$


Proof Computing $Q-P$, we have

$$
\begin{aligned}
Q-P= & {\left[n K_{t}^{s} K_{s}^{r} K_{r}{ }^{t}-\frac{2(n+1)}{n+2} K K_{j i} K^{j i}+\frac{1}{n+2} K^{3}\right] } \\
& -\left[n K_{t}^{s} K_{s}^{r} K_{r}{ }^{t}-\frac{2 n-1}{n-1} K K_{j i} K^{j i}+\frac{1}{n-1} K^{3}\right] \\
= & \frac{3 n K}{(n-1)(n+2)}\left[K_{j i} K^{j i}-\frac{K^{2}}{n}\right] \\
= & \frac{3 n K}{(n-1)(n+2)}\left(K_{j i}-\frac{K}{n} g_{j i}\right)\left(K^{j i}-\frac{K}{n} g^{j i}\right),
\end{aligned}
$$

which proves Lemma 5.

Lemma 6. In a Riemannian manifold whose Ricci tensor is positive semi-definite, we have $P \geqq 0$.

Proof By the assumption, we have $0 \leqq \lambda_{1} \leqq \lambda_{2} \leqq \cdots \leqq \lambda_{n}$. Take arbitrarily three indices $i, j$ and $k$ such that $i<j<k$. Then we have

$$
\begin{aligned}
& \lambda_{i}\left(\lambda_{i}-\lambda_{j}\right)\left(\lambda_{i}-\lambda_{k}\right)+\lambda_{j}\left(\lambda_{j}-\lambda_{i}\right)\left(\lambda_{j}-\lambda_{k}\right)+\lambda_{k}\left(\lambda_{k}-\lambda_{i}\right)\left(\lambda_{k}-\lambda_{j}\right) \\
& =\lambda_{i}\left(\lambda_{i}-\lambda_{j}\right)\left(\lambda_{i}-\lambda_{k}\right)+\left(\lambda_{j}-\lambda_{k}\right)^{2}\left(\lambda_{j}+\lambda_{k}-\lambda_{i}\right) \geqq 0 .
\end{aligned}
$$

Thus we have, using (3.9), $P \geqq 0$.

\section{$\S 4$. Proof of the theorem}

Assume that, in a Kaehlerian manifold $M$ of dimension $n$, the scalar curvature is constant, the Bochner curvature tensor vanishes and the Ricci tensor is positive semi-definite. Then we have $K \geqq 0$, and consequently, by Lemmas 3,5 and 6 , we have

$$
\begin{aligned}
& \frac{1}{2} \Delta\left(K_{i h} K^{i n}\right) \\
& =\frac{1}{n+4}\left[P+\frac{3 n K}{(n-1)(n+2)}\left(K_{j i}-\frac{K}{n} g_{j i}\right)\left(K^{j i}-\frac{K}{n} g^{j i}\right)\right] \\
& +\left(\nabla_{j} K_{i n}\right)\left(\nabla^{j} K^{i n}\right) \geqq 0 .
\end{aligned}
$$

Therefore, if we assume that $M$ is compact, then we have $\Delta\left(K_{i n} K^{i n}\right)$ $=0$, from which and (4.1), if $K \neq 0$, we have

$$
K_{j i}=\frac{K}{n} g_{j i},
$$

that is, $M$ is an Einstein manifold. Thus, by Lemma 2, the Kaehlerian manifold $M$ is of constant holomorphic sectional curvature.

If $K=0$, then we have 


$$
\lambda_{1}=\lambda_{2}=\cdots=\lambda_{n}=0
$$

because of $\lambda_{1}=\lambda_{2}=\cdots=\lambda_{n} \geqq 0$ and consequently

$$
K_{j i}=0 \text {, }
$$

from which

$$
L_{j i}=0, \quad M_{j i}=0 .
$$

Thus, the Bochner curvature tensor being zero, the curvature tensor $K_{k j i}{ }^{h}$ vanishes, and consequently the Kaehlerian manifold $M$ is of zero curvature.

Thus the universal covering manifold of $M$ is a complex projective space $C P^{n / 2}$ or a complex space $C^{n / 2}$. Thus the theorem stated at the end of the introduction has been completely proved.

Tokyo Institute of Technology

\section{Bibliography}

[1] T. AUBin: Sur les variétés kählériennes compactes à courbure scalaire constante, C. R. Acad. Sci. Paris, 266 (1968), 422-423.

[2] S. Bochner: Curvature and Betti numbers, II, Annals of Math., 50 (1949), 77-93.

[3] S. I. GOLDBERG : On conformally flat spaces with definite Ricci curvature, Kōdai Math. Sem. Rep., 21 (1969), 226-232.

[4] P. J. RYAN: A note on conformally flat spaces with constant scalar curvature, to appear.

[5] S. TAchibana: On the Bochner curvature tensor, Natural Science Report, Ochanomizu University, 18 (1967), 15-19.

[6] M. TANI: On a conformally flat Riemannian space with positive Ricci curvature, Tôhoku Math. J., 19 (1967), 227-231.

[7] K. YANo: Differential geometry on complex and almost complex spaces, Pergamon Press, 1965.

[8] K. Yano: On complex conformal connections, to appear in Kōdai Math. Sem. Rep.

[9] K. YANo and S. Bochner: Curvature and Betti numbers, Annals of Math. Studies; No. 32, 1953.

(Received January 6, 1974) 